\title{
A LINEAR ACTIVE DIPOLE ARRAY LOADED WITH NEGATIVE RESISTANCE DEVICES
}

\author{
Chien-Chang Huang and Tah-Hsiung $\mathrm{Chu}^{*}$ \\ Electrical Engineering Department \\ National Taiwan University \\ Taipei, Taiwan, Republic of Chinit
}

\begin{abstract}
Analyses of a linear active dipole array loaded with negative resistance devices under injection-locked arrangement are presented. In the arrangement, each dipole loaded with an active diode is treated as a microwave source, and an external injection plane wave illuminates the active dipoles to sychronize the linear active array. Analysis is based on the method of moments of dipoles and the stability analysis of injection-locked oscillators. Simulation results yield the array radiation field intensity and locking range for various incident field strength. Variation of loaded diode chartacteristics on the array performance are also considered.
\end{abstract}

\section{Introduction}

Solid-state microwave sources have several advantages over tubes due to their size, life time and small power consumption. The limited output power level, however, makes them not practical for many applications. Combining solidstate devices to generate high output power then becomes attractive. Since the loss of guide-wave type power combiner reduces its efficiency in the microwave and millimeter wave regions, the quasi-optical power combining techniques have been proposed experimentally [1]-[3] by combining the radiation field of each microwave source through antennas in the spatial domain. However, their simulation models did not include the nonlinear effect of active devices.

The purpose of this study is to present quantitative analysis of an active dipole array under injection locking. In the modelling, linear dipole antennas and two terminal negative resistance active devices (for example, Gunn diode) are considered. First, each dipole antenna is treated as an equavalent circuit using the method of moments. A nonlinear circuit equation is then construced to include the injection-locked oscillator (ILO). Due to the nonlinearity, multivalues of its output power are obtained, and stability criteria are derived to study the stability properties of the injection-locked dipole antenna. Numerical examples show the array radiation field intensity and locking range under different injection wave strength. The radiation patterns of uniform or different characteristics of actve devices in the array are also giren.

\section{Analysis}

Consider a linear dipole array loaded with negative resistance devices as in Fig.1(a). If the incident wave field strength is large erough, the mutual coupling effect of dipole array can be neglected [4]. The equavalent circuit of each element is expressed using the method of moments as in Fig.1(b), where $Y_{\text {in }}$ is the dipole input admittance and $I_{e q}$ is the short-circuit current induced by the incident field $E_{i n}$. The active two-terminal device: is modeled to include 


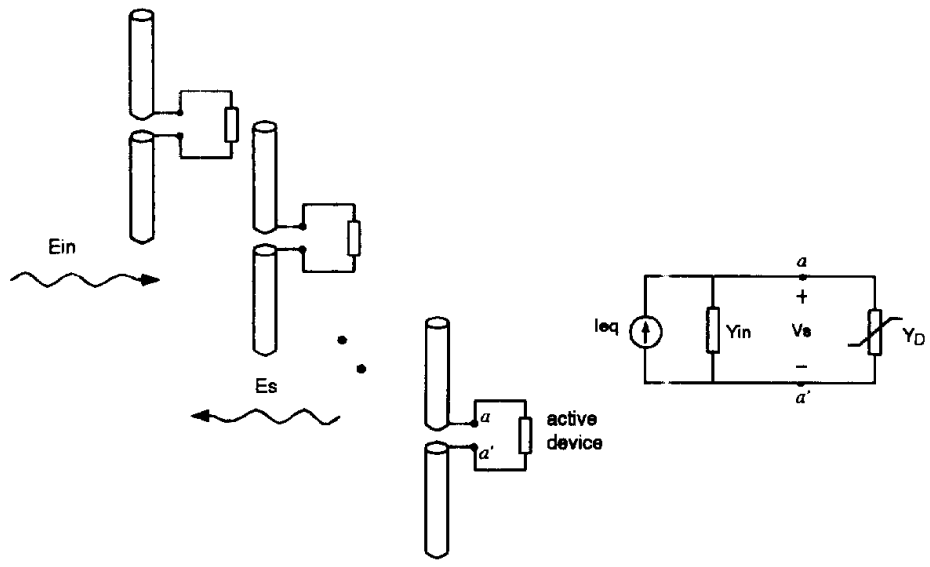

(a)

(b)

Figure 1: (a) A linear active dipole array loaded wilh negative resistance devices and (b) its equivalent circuit of each element.

the third order nonlinearity as [5]

$$
Y_{D}=G_{n 0}+G_{n 2} V^{2}
$$

As $Y_{i n}+Y_{D}=0$ and $I_{e q}=0$, the dipole may radiate without incident wave, i.e., in the free-running oscillation state. The operating freq 1ency $\omega_{o}$ and terminal voltage $V_{s o}$ of each dipole can then be solved. As an external incident wave $E_{\text {in }}$ with frequency $\omega_{\text {in }}$ illuminats the dipole array to sychronize each dipole elements, the injection-locked oscillator (ILO) can ther be constructed. The circuit equation becomes

$$
\left(Y_{\text {in }}+Y_{D}\right) V_{\imath} e^{j \phi}=I_{\text {eq }}
$$

Solving $V$, in eq. (2), it is found that there may be multi-values including false solutions. To evaluate the stability properties of each solution, one can use the first-order approximation of the total admittance expansion with respect to $\omega_{i n}$, and perturb the variables $V_{\text {. }}$. The stable condition for the injection-locked oscillator becomes [5]

$$
\begin{aligned}
& 2\left(G_{T} \frac{\partial B_{T}}{\partial \omega}-B_{T} \frac{\partial G_{T}}{\partial \omega}\right)+\left(\frac{\partial G_{T}}{\partial V_{s}} \frac{B_{T}}{\partial \omega}-\frac{\partial B_{T}}{\partial V_{s}} \frac{\partial G_{T}}{\partial \omega}\right) V_{\imath}>0 \\
& G_{T}\left(G_{T}+\frac{\partial G_{T}}{\partial \omega} V_{\bullet}\right)+B_{T}\left(B_{T}+\frac{\partial B_{T}}{\partial V_{s}}\right)>0
\end{aligned}
$$


where $G_{T}$ and $B_{T}$ are the total conductance and susceptance respectively. Based on eqs.(3), (4) the locked state can be determined by checking the stability properties of each possible value $V_{s}^{2}$ under the injection condition.

\section{Numerical Examples}

In the simulation example, each dipole is with $10 \mathrm{~cm}$ long and $0.135 \mathrm{~cm} \mathrm{di-}$ ameter, and the diode parameters $G_{n 0}, G_{n 2}$ are -0.04 and 0.24 . For single active dipole element calculation, the free-running frequency is $1.40523 \mathrm{GHz}$ and $V_{o o}^{2}=0.10948 V^{2}$. At the injection condition, Fig.2(a) shows the stability diagram with normally incident plane wave $E_{i n}=10.0 \mathrm{mV} / \mathrm{m}$. Note the solution is shown unstable under the stability boundary curve. The locking range corresponds to the frequency interval covered by the oval-shaped line.

Fig.2(b) shows the incident and radiated field strength. For small injection field strength, the radiated field strength is almost unchanged because of the free-running power dominant. The ILO acts as an amplifier as a larger injection field applied, and causes the radiated field strength increases drastically.

A 7-element linear active dipole array is considered with uniform or different diode characteristics for the array simulation. Fig.3(a) shows the array locking range for various incident field strength. The locking range decreases a little as the device parameters are simulated with normal distribution. The resulting array radiation patterns are shown in Fig.3(b). It can be observed that they differ slightly at the sidelobe levels.

\section{Conclusion}

In this study, an approach based on the moment method and stability analysis is presented to simulate a linear active array under injection locking. The array locking range and output radiation field strength are calculated numerically. Simulation results indicate that variation of active device parameters may reduce the locking range slightly, but the radiation pattern is almost unchanged.

\section{References}

[1] J. W. Mink, "Quasi-optical power combining of solid-state millimeterwave sources," IEEE Trans. Microwave Theory Tech., vol. MTT-34, pp. 273-279, Feb. 1986.

[2] R. A. York and R. C. Compton, "A 4 multiplied by 4 active array using Gunn diodes," IEEE AP/S Symposium, pp. 1146-1149, 1990.

[3] Z. B. Popović, R. M. Weikle II, M. Kim and D. B. Rutledge, "A 100MESFET planar grid oscillator," IEEE Trans. Microwave Theory Tech., vol. MTT-39, pp. 193-200, Feb. 1991.

[4] J. Birkeland and T. Itoh, "A 16-element quasi-optical FET oscillator power combining array with external injection lockirg," IEEE Trans. Microwave Theory Tech., vol. MTT-40, pp. 475-481, Mar. 1992.

[5] G. H. B. Hasson and K. I. Lundström, "Stability criteria for phase-locked oscillators," IEEE Trans. Microwave Theory Tech., vol. MTT-20, pp. 641645, Oct. 1972. 


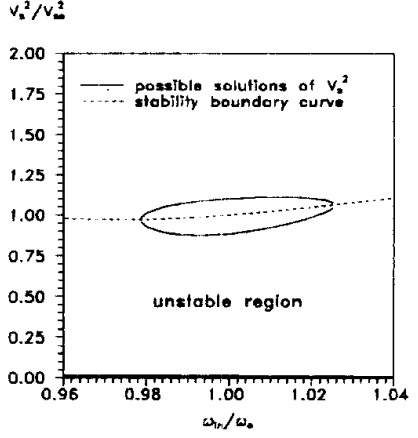

(a)

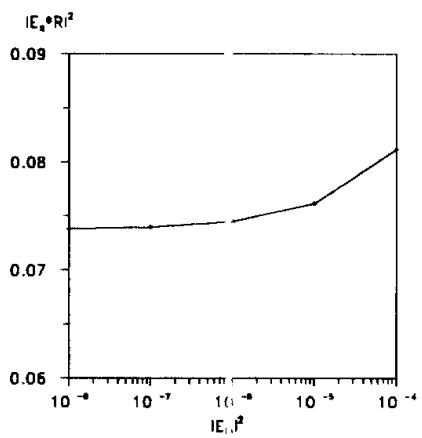

(1)

Figure 2: (a) Stability diagram of a single active dipole antenna where $E_{i n}=10 \mathrm{mV} / \mathrm{m}$ and (b) the scattered field for various incicient wave strength.

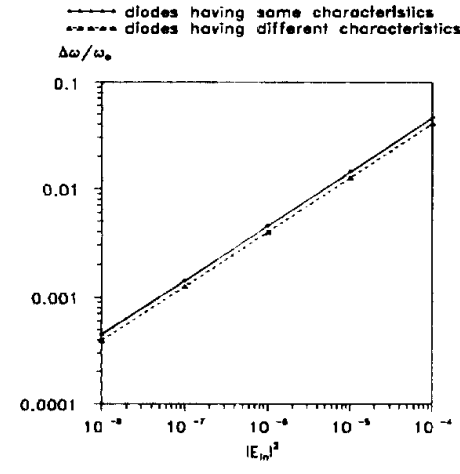

(a)

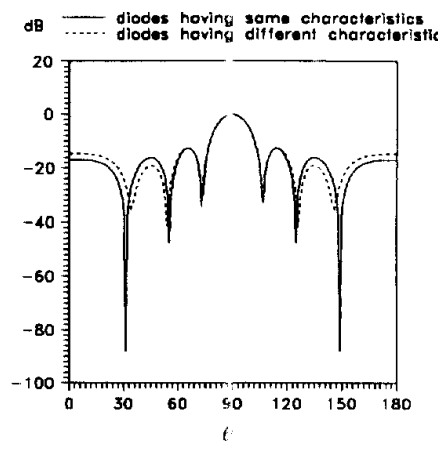

(li)

Figure 3: (a) The locking range of the linear active dipole array for various incident wave strength and (b) the radiation pattern of the array at $E_{\text {in }}=1.0 \mathrm{mV} / \mathrm{m}$, where uniform and different loaded diode characteristics are shown. 